\title{
A Multicenter Point Prevalence Survey of Antibiotic Use in Punjab, Pakistan: Findings and Implications
}

Running Title: A Multicenter Point Prevalence Survey of Antibiotic Use

Zikria Saleem ${ }^{1,2}$, Mohamed Azmi Hassali ${ }^{1}$, Ann Versporten ${ }^{3}$, Brian Godman ${ }^{4,5,6}$, Furqan Khurshid Hashmi ${ }^{7}$, Herman Goossens $^{3}$, Fahad Saleem ${ }^{8}$

${ }^{1}$ School of Pharmaceutical Sciences, Universiti Sains Malaysia, Malaysia

${ }^{2}$ Rashid Latif College of Pharmacy, Pakistan

${ }^{3}$ Laboratory of Medical Microbiology, Vaccine and Infectious Disease Institute, Faculty of Medicine and Health Sciences, University of Antwerp, Belgium

${ }^{4}$ Department of Clinical Pharmacology, Karolinska Institute, Stockholm, Sweden

${ }^{5}$ Strathclyde Institute of Pharmacy and Biomedical Sciences, Strathclyde University, Glasgow, UK

${ }^{6}$ Health Economics Centre, University of Liverpool Management School, Liverpool, UK

${ }^{7}$ University College of Pharmacy, University of the Punjab, Lahore, Pakistan

${ }^{8}$ Faculty of Pharmacy and Health sciences, University of Balochistan, Quetta, Pakistan

\section{Corresponding Author}

Zikria Saleem, School of Pharmaceutical Sciences, Universiti Sains Malaysia, Malaysia

Phone Number: +92322-9801981; Fax No: +92-42-99211624. Email ID: xikria@gmail.com

(Accepted for publication Expert Review Anti-Infective Therapy - Please keep CONFIDENTIAL)

\begin{abstract}
:
Objective: In line with the recent global action plan for antimicrobial resistance, the first time such a comprehensive survey has been undertaken in Pakistan, sixth most populous country.

Method: This point prevalence survey (PPS) was conducted in 13 hospitals among 7 different cities of Pakistan. The survey included all inpatients receiving an antibiotic on the day of PPS. A web-based application was used for data entry, validation, and reporting as designed by the University of Antwerp.
\end{abstract}


Results: Out of 1954 patients, 1516 (77.6\%) were treated with antibiotics. Top three most reported indications for antibiotic use were prophylaxis for obstetrics or gynaecological indications (16.5\%) and gastrointestinal indications $(12.6 \%)$ and lower respiratory tract infections (12.0\%). Top three most commonly prescribed antibiotics were ceftriaxone (35.0\%), metronidazole (16.0\%) and ciprofloxacin (6.0\%). Out of total indications, $34.2 \%$ of antibiotics were prescribed for community-acquired infections (CAI), 5.9\% for healthcare-associated infections (HAI), and $57.4 \%$ for either surgical or medical prophylaxis. Of total surgical prophylaxis, $97.4 \%$ of antibiotics were given for more than one day.

Conclusion: Study concluded that unnecessary prophylactic antibiotic use is extremely high and broad-spectrum prescribing is common. There is a considerable need to work on a national action plan of Pakistan on antibiotic resistance.

Keywords: Point Prevalence Survey, Antimicrobial Prescribing, Antimicrobial Resistance, Hospitals, Pakistan

\section{INTRODUCTION}

Infectious diseases were the major cause of death in humans until 1911 when the first antibiotic agent was introduced as a "magic bullet" [1,2]. Since their development, the significant role of antibiotics in saving lives has been evident [3]. However, all these benefits are related to their rational use [4]. Unfortunately, the irrational use of antibiotics increases the risk of development of antimicrobial resistance (AMR), increasing morbidity, mortality and costs, with costs including additional visits to emergency departments, extra prescriptions for adjuvant therapies as well as prolonged hospital stay, leading to worldwide calls to address this [5-13]. For example, colistin has re-emerged as a valued antibiotic despite its side-effects because of the emergence of carbapenem-resistant gram-negative bacteria $[14,15]$. This has resulted in a number of countries, including South Africa, to develop programmes to limit its use to preserve its effectiveness [16], with colistin also now included in the WHO reserve list of antibiotics [17]. According to the Council for Appropriate and Rational Antibiotic Therapy (CARAT), criteria for optimized antibiotic therapy include therapeutic safety and benefits, evidence-based results, cost-effectiveness and the optimal antibiotic for the optimal duration [18]. Appropriate dosing regimens should be adopted by following guidelines for antibiotic prescribing $[18,19]$.

Data on antibiotic use in healthcare settings in Pakistan are currently scarce. We are aware of the excessive use of antibiotics in ambulatory care [20-22]. However, only a few published studies are available regarding the pattern of antibiotic use in Pakistan in hospitals [23,24] including their irrational use [25]. We are also aware that there have 
been initiatives to try and improve antibiotic use using antibiotic stewardship programmes (ASP) in Pakistan [26]. Contrary to other countries and continents including Africa, Americas, Asia, Australia, Europe, Kenya, and Turkey [27-36], no point prevalence survey (PPS) to evaluate antimicrobial use and resistance has so far been conducted in Pakistan. This is important to address concerns with the irrational use of antimicrobials in hospitals in Pakistan. Consequently, the main objectives of this multicenter PPS were to evaluate the prevalence of antibiotic use among hospitals in Pakistan and to describe the current pattern of antibiotic prescribing by type of patient's characteristics, healthcare facilities, and specialties. This builds on our recently published study regarding the prevalence of hospitalacquired infections in Pakistan [37]. The findings will help inform future strategies to improve antibiotic prescribing among hospitals in Pakistan.

\section{METHODOLOGY:}

\subsection{Study Design:}

A multicenter PPS of antibiotic use was conducted using the Global-PPS method [36,38]. This standardized survey method was used to document and evaluate antimicrobial prescribing patterns at hospital, ward and patient level.

\subsection{Study Settings:}

The study setting for this first PPS study in Pakistan was Punjab. Punjab was chosen as the study region because it is the most populous province of Pakistan. A complete list of all public, private and charity hospitals of Punjab were acquired from the Director General Health Services office, Department of Health, Government of the Punjab, Pakistan. The hospitals from public, private and charity hospital groups from the different cities in Punjab, Pakistan were invited to participate in this survey. In case of refusal of the first selected hospital, the next health care setting on the list was selected. Participation of the hospital was voluntary. Thirteen hospitals from geographically and economically different cities of Punjab agreed to participate in the survey, which provided a representative insight into current antimicrobial prescribing practices. The health care facilities providing only nursing care, rehabilitation centers or psychiatric centers were not included in line with other PPS studies [36,39].

\subsection{Instrument of Measure:}

Standardized paper data collection forms were used to collect data at each hospital, ward and patient level. The hospital data form included the type of the hospital, departments, number of hospitalized patients, single-room bed capacity and total bed capacities of the surveyed hospital. A review of the availability of antibiotics in the hospital formulary 
was checked before data collection. The Ward data form included the department specialty, total bed capacity and the total number of admitted patients (denominator data). The Patient data form included the age, gender, reason for antibiotic prescribing, the prescribed antibiotics dosage regimen, the antibiotic prescription ratio active, and the causative microorganisms. For the dose of a combination antimicrobial, the sum of each antibiotic substance excluding the enzyme inhibitors was recorded. A set of quality indicators included whether the reason for the prescription and a stop/review date was written in the notes; and whether the antibiotic was prescribed according to the guidelines if this existing, with the latter increasingly indicative of quality prescribing [36,40,41]. A web-based application was used for data-entry, validation, and reporting as designed by the University of Antwerp [36,38].

\subsection{Inclusion criteria:}

All in-patients that stayed overnight and remained in the ward at 08:00 am on the day of the survey were included. Data were collected only from those patients who were receiving at least one antimicrobial for at least one clinical treatment condition or prophylaxis at the time of the survey. Beside antibacterials for systemic use, the survey included also antimycotics, antifungals and antivirals for systemic use infections in more than one site in the same patient were reported as separate infections.

\subsection{Exclusion criteria}

Short stay patients that did not stay overnight, those discharged patients before 08.00 am on the day of the survey that were waiting for transport to their dwelling, patients admitted after 8am on the day of the survey, patients staying in long-term care units including dialysis centers and patients in emergency and outpatient departments were not included.

\subsection{Data Collection:}

The data were collected using a structured data collection tool [36,38]. All patients admitted on the ward at 08.00 am on the day of the survey were counted in the denominator. Whereas, all inpatients on antimicrobials at 08.00 am were included in the numerator, and forms were completed for these patients only. All the prescribed antimicrobials at the time of the survey were included. Patients were included as part of the initial ward of admittance if transferred to another ward after 8.00 a.m. All neonates born before 08.00 am on the day of the survey were also included. The surgical wards were not surveyed on a day following a holiday but on the other days of the week in order to capture 
information about prophylaxis in the last 24 hours [36]. Surgical prophylaxis included prophylactic agents to prevent surgical site infections. Long-acting antibiotics and intermittent treatment on alternative days were also included if given 24 hours before the survey. Medical prophylaxis was defined as the use of antibiotics to prevent infections in patients with medical conditions [36]. Infections were considered as community-acquired infections (CAIs) if symptoms started $<48$ hours from admission to hospital (or present on admission), and hospital-acquired infections (HAIs) if symptoms started 48 hours after admission [36]. The last prescribed antibiotic was recorded if the antibiotic prophylaxis or treatment was changed on the day of the survey before or at $08.00 \mathrm{am}$. Additional details of the variables from the patient's medical case notes and prescribing charts were recorded after discussions with nursing staff and physicians if pertinent. However, in most cases, only the patients' notes were reviewed in order to gain a good understanding of the current situation especially if crucial data was left out such as the rationale for antibiotic selection. The data were double checked for completeness and accuracy in order to rule out missing or inconsistent data. There was no contact with any patient at any moment. All the data were entered onto the web-based Global-PPS application [38]. Data from all wards of a single hospital were collected within 2-4 weeks. In order to minimize the effect of movement of patients between wards and within the hospital, each ward was completely surveyed within one day. The process of data collection was completed between October 2017 and February 2018. The Anatomical Therapeutic Chemical (ATC) classification system of the WHO was used to classify the different antibiotics used [42].

\subsection{Ethical Considerations:}

Application for Ethical clearance prior to the conduct of the study was sought from the Human Ethics Division of University College of Pharmacy, University of the Punjab, Lahore (HEC/1000/PUCP/1925B). Subsequent approval to conduct the study in the identified hospitals was obtained from the hospital management. All collected data were anonymized during the time of data collection.

\subsection{Statistical Analysis}

Data were analyzed using the latest versions of Microsoft Excel and SPSS (version 22 IBM, California, USA). On categorical variables, descriptive statistics (frequency, percentages, mode, and medians) were applied. Continuous variables were presented with median and range. 


\section{RESULTS:}

A total of 13 hospitals with 3074 beds were surveyed in this prevalence study from seven different cities of Pakistan. The survey was conducted in five private and eight public sector hospitals including secondary, tertiary and specialized health care facilities. Basic patient data from the different hospitals treated with antimicrobials is presented in Table 1. During this PPS, 1954 (63.6\% bed occupancy) patients with a median age of 35 years were hospitalized on the day of the survey. Out of these, 1516 patients $(77.6 \%)$ were treated with one or more antimicrobials of which 1503 patients received at least one antibiotic for systemic use $(76.9 \%$; ATC code J01). There was an appreciable variation in antibiotic use prevalence across the hospitals, ranging from 50.9\%-100\% of all patients in the hospital.

The total number of prescribed antimicrobials was 2483 (J01; 96.6\%, J02; 0.4\%, J04; 0.8\%, J05; 0.8\%, P01; 0.6\%, A07; 0.7\%) (Table 2), of whom 1177 (47.7\%) were given to male patients and 1306 (52.6\%) were given to female patients. The average number of antimicrobials prescribed per patient (1.64) ranged from 1.14 to 1.97 antimicrobials per patient. Nine hundred and sixty-one (38.7\%), 1,404 (56.5\%) and 118 (4.8\%) antibiotics were prescribed in surgical departments, medical departments, and ICU, respectively (Table 1). Parenteral (91.5\%) antibiotic use was very prevalent. Of the total prescribed antibiotics, 55.5\% were prescribed to patients in the age group 25-65 years, followed by $26.3 \%, 8.9 \%, 6.5 \%$ and $2.7 \%$ to patients in the age groups $1-25$ years, above 65 years, 1 month-1 year and less

than 1 year respectively (Appendix 1). The admitted patients were more likely to receive antibiotics as prophylaxis (57.4\%). Of the total 1,426 antibiotics for prophylactic use, 893 (62.6\%) were used for surgical prophylaxis whereas $533(37.4 \%)$ were prescribed for medical prophylaxis. Most of the antibiotics for surgical prophylaxis were given for more than one day $(97.4 \%)$. For the management of infections, $850(85.3 \%)$ antibiotics were given for CAIs whereas 147 (14.7\%) were given for HAIs.

In the majority of cases, the reasons for prescribing the various antibiotics were not mentioned in the patient's medical file (76.2\%). Only 57 culture reports were found at the time of the survey as most of the antibiotics were prescribed empirically (96.2\%). Fifty-eight different types of antibiotics were used, but just two (ceftriaxone and metronidazole) comprised $51 \%$ of all antibiotic treatment. Most of the hospitals were using 20 different types of antibiotic substances, ranging from 9-30. Of the total number of antibiotics prescribed, 772 (50.9\%) patients were prescribed one antibiotic, $558(36.8 \%)$ were prescribed two antibiotics and $186(12.3 \%)$ patients were prescribed three or more antibiotics. 
Most of the surveyed patients were admitted into the general medical wards (25.0\%), surgical wards (18.4\%) and obstetrics and gynecology wards (14.4\%). Other than these wards, all other wards were also surveyed as per the hospital specialty. The details about the use of the different antimicrobials in the different clinical wards and medical indications are summarized in Tables 3 and 4 . Appendixes 2 to 4 provide details at the hospital level. Obstetric or gynecological prophylaxis $(16.5 \%)$, gastrointestinal prophylaxis $(12.6 \%)$, lower respiratory infections $(12.0 \%)$ and general medical prophylaxis $(10.5 \%)$ were the most common clinical indications for antibiotic use.

The top three most commonly prescribed antibiotics were ceftriaxone $(35.0 \%)$, metronidazole (16.0\%) and ciprofloxacin (6.0\%) (Table 3). Ceftriaxone remained the most used antibiotic for different treatments or prophylactic use, ranging from on average $12.7 \%$ of use in the ICU to on average $40.4 \%$ of use in the medical departments (Table 4). In addition to these top three commonly used antibiotics, there was a relatively high use of amikacin (11.0\%), meropenem (10.2\%), piperacillin and enzyme inhibitor (10.2\%) and vancomycin (9.3\%) was observed among ICU patients compared to other departments. Metronidazole IV was highest for surgical prophylaxis (26.7\%). Ampicillin was most frequently used in neonates $(8.8 \%)$ and infants $(20.8 \%)$. Moxifloxacin was frequently prescribed among patients aged 25-65 years (4.4\%) and age above 65 years (9.0\%). It proved impossible to assess the quality of prescribing as currently there are no accepted national guidelines giving advice on antimicrobial prescribing [43].

\section{DISCUSSION:}

Effective surveillance of antibiotic use is invaluable to help rationalize antibiotic prescribing, thereby helping control the emergence of multidrug resistance microbes, observing the efficiency of policies, identifying targets for quality improvement and informing the policymakers $[7,9,28,44,45]$. The global threat of increasing AMR prompted us to conduct this first point prevalence survey among selected hospitals of Pakistan [10]. In the present study, 77.6\% of admitted patients on one single day had consumed at least one antimicrobial. This is much higher than prevalence rates reported in other PPS studies across the world including the Global PPS (34.4\%), Europe (27.4\% - 34.4\%\%), Asia including China (37.2\% - 55.6\%), North America (38.6\% - 50.0\%) and Turkey (30.6\%) [28,32-34,36,44]. However, this is similar to some of the hospitals seen in the Global PPS study, particularly the African countries, as well as Botswana and Kenya [36,46,47] but not Ghana (51.4\%) [48]. In Botswana, more than two-thirds of admissions in their PPS study were due to infectious diseases with $40 \%$ of those tested were HIV positive [47]. In addition, our results are comparable with other hospitals in China (75.3\%) [49], which has already resulted in the instigation of antibiotic stewardship strategies in China to address antibiotic overuse [50-52]. Among the identified regions 
worldwide, the highest use of antibiotics was seen in intensive care units (ICU) of Vietnam (84.6\%) among the adult population [53]. In the Global PPS, up to $69.7 \%$ of patients in ICU were prescribed an antibiotic [36], similar to the findings in Kenya [46]. We also saw a high use of antibiotics in ICU.

There was also a high use of antibiotics for medical prophylaxis in our study (37.4\% of $57.4 \%$ of patients who received an antimicrobial). This is similar though to the situation seen in Kenya ( $29 \%$ of antimicrobial use) but much lower than seen in the Global PPS (7.4\%) [36,46]. The reasons for this high rate are currently unknown; however, this will be investigated further to reduce inappropriate antimicrobial prescribing. Approximately $50 \%$ of patients were prescribed two or more antibiotics in our study, which is similar to a study conducted in America [33]. We believe this high rate of combination antibiotic treatment is needed to increase the spectrum of antibiotics prescribed without the support of a culture report. This is also a concern that needs to be addressed, and we will be pursuing this in future projects. In the present study, the most commonly prescribed antibiotic was ceftriaxone (35\%) which is comparable to a study from China [49], with ceftriaxone included in the WHO list of Access antibiotics [17]. In other regions, however, such as in 183 hospitals of the USA, the prevalence of ceftriaxone use was only $10.8 \%$ [33]. The majority of antibiotics prescribed were also on the WHO list of Access antibiotics (Table 3), with moxifloxacin, piperacillin with an enzyme inhibitor and cefoperazone on the Watch list [17]. Their use will be the subject of future research projects.

In the present study, ceftriaxone was also commonly prescribed for surgical prophylaxis, similar to a number of regions in the Global PPS particularly Africa as well as in Kenya for surgical prophylaxis $[36,46]$. The routinely use of ceftriaxone for prophylaxis in our study is of concern as it should not routinely be used for prophylaxis [54]. Inappropriate ceftriaxone use can lead to the emergence of multidrug-resistant strains if not addressed $[55,56]$. There is also concern that antibiotics for surgical prophylaxis were given for more than one day (97.4\%), which is against current guidance as this can increase AMR rates [57,58]; however, this is similar to the situation seen in other countries $[36,46,59]$. The threat of surgical site infections should not be ignored while following guidelines $[60,61]$. A number of studies have highlighted that even in the most documented evidence-based area of practice, physicians are inclined to prescribe antibiotics for more than 24 hours [62]. As a result, physicians should be encouraged to write the reason to start antibiotic treatment, document the start date and follow up the duration of use in the patient's medical notes. 
This will be followed up in future studies. The high use of ceftriaxone in this study also suggests cheap, easy or free availability and affordability, which needs to be researched further [63].

As mentioned, there are almost no local or national guidelines or recommendations for empiric antibiotic use in hospitals in Pakistan [43]. Our results suggest the urgent need to develop these starting with guidelines for the most common indications as well as for surgical and medical prophylaxis. This could be part of an ASPs to be developed at a national level, and could also be part of the national action plan of Pakistan for AMR [64]. The UK, for example, has successfully managed to reduce the use of the third-generation cephalosporins by introducing antimicrobial stewardship programs $[65,66]$, providing guidance to others. In addition, a lower proportion of broad-spectrum antibiotics indicates better practice [67]. It is also important that ASP also include quality targets, a timeline as well as a dedicated budget to achieve desired goals. However, it is acknowledged that it is more challenging introducing ASPs in LMICs due to a number of issues including manpower challenges [68].

Variations in antibiotic use patterns may also be due to differences in the spectrum of diseases and the facilities across countries and regions; however, this needs to be investigated further before any definitive statements can be made. There is also likely to be variation among the departments in the pattern of antibiotic use within hospitals in Pakistan. For example, large and specialized hospitals have more immunocompromised or severely injured and ill patients, or those in need of complex surgical procedure, as compared to small hospitals affecting utilization patterns [31]. Encouragingly, the use of quinolones in the present study is comparable to most of the European countries but less than those reported in France $[31,69,70]$. Overall, the extensive use of broad-spectrum antibiotics in Pakistan could be explained by high bacterial resistance rates [71], and the potential for practitioners to overprescribe antibiotics if they view such practices as a viable way to ensure a speedy recovery [72,73]. Additionally, physicians in Pakistan may be influenced by pharmaceutical companies if they are the principal source of information as seen in other settings in Pakistan and other countries [21,74-76].

Another concern is the very high usage (91.5\%) of parenteral antibiotics observed in this study as compared to previously published PPS studies [45]. We believe this goes hand in hand with the high use of third-generation cephalosporins (ceftriaxone) for which no oral equivalent is currently available. Nevertheless, many physicians and patients consider that parenteral therapy is superior and more effective than oral administration [77]. Moreover, the use of parenteral antibiotics is inevitable in case of life-threatening infections, the age of the patient especially infants, 
and the availability of dosage form. Whenever possible though, each hospital should develop individual guidelines to switch from intravenous to oral treatment in view of the several clinical and economic benefits associated with timely conversion to oral from intravenous therapy $[78,79]$. In our study, physicians of only one specialized cancer care hospital were found to prescribe fewer antibiotics (50.9\%) as per the advice of pharmacists. The literature supports the fact that interventions of a pharmacist by interacting directly in the ward with physicians can play an important role in optimizing antibiotic prescribing, leading to a decrease in health care costs and days of hospitalization $[80,81]$. This will also be followed up in future studies.

Finally, as seen, prophylactic antibiotic use without culture reports was high, relatively much higher in smaller hospitals of those surveyed. This reflects the general lack of reporting the rationale for antimicrobial use. As a result, as mentioned, physicians should be encouraged to write the reason to start antibiotic treatment in the patient's medical notes. The present study has a number of limitations. Firstly, it was impossible to assess the appropriateness of antibiotic therapy in terms of choice and duration. Nonetheless, surveillance is the first step in identifying, appraising and progressing the existing situation with respect to inappropriate antibiotic prescribing. Secondly, a number of patient's medical notes were not sufficient to evaluate the diagnosis of infection. This may have underestimated or overestimated infection rates. Thirdly, as this was the first PPS in these hospitals, sequential surveys should be conducted to reveal any seasonal changing epidemiology among the hospitals [82]. Lastly, because participation of hospital was solely voluntary, data cannot be generalized to the whole of Pakistan because only a few hospitals of one Province participated in the survey. In spite of the above-mentioned limitations, the present survey provides insight into the prevalence and pattern of antibiotic use in Pakistan. The outcomes of this survey highlight the significance of surveillance of antibiotics and the need of antibiotic policies to enhance the appropriate use of antibiotics in the future. The findings of this survey could be the basis for planning future goals for appropriate management of antibiotic use and antibiotic stewardship including developing pertinent quality indicators based on previous studies $[36,40]$. The important features of this methodology are its ease of use and cost-effectiveness.

\section{CONCLUSION AND RECOMMENDATIONS:}

There is considerable cause for concern regarding the current use of antibiotics in hospitals in Pakistan. Consequently, there is an urgent need to start working on a national action plan in Pakistan in order to rationalise the use of antibiotics. Stewardship efforts, in particular, should be focused on the use of ceftriaxone especially any prophylactic use, the 
length of prophylaxis and the reasons for antibiotic prescribing in the first place. In addition, the rapid conversion to oral antibiotics should be implemented where pertinent. To preserve the future effectiveness of antibiotics and reduce patient harm due to AMR, it is imperative to rationally scrutinize and improve the prescribing practices. The study is

a first step to provides an insight into antibiotic use among Pakistani hospitals and highlights directions for improvement of antibiotic prescribing quality. Taken together, a multifaceted policy that includes regulation, collaboration, education, and increased financial support is required at the national level in Pakistan. Future studies can also be part of the Global PPS study to enhance comparisons with other LMICs.

\section{Acknowledgments}

This study would not have been possible without the contribution and involvement of administration and staff in all participating Pakistani hospitals. Their cooperation is thankfully appreciated.

\section{Financial support}

This paper was not funded.

\section{Conflict of interest}

The authors declare that there are no actual or potential conflicts of interest in relation to this article.

\section{References}

*of importance, ** of considerable importance

1. Zaffiri L, Gardner J, Toledo-Pereyra LH. History of antibiotics. From salvarsan to cephalosporins. Journal of Investigative Surgery, 25(2), 67-77 (2012).

2. Nicolaou KC, Rigol S. A brief history of antibiotics and select advances in their synthesis. The Journal of antibiotics, 71(2), 153 (2018).

3. Hamilton KW. Miracle Cure: The Creation of Antibiotics and the Birth of Modern Medicine. Emerging infectious diseases, 25(1), 196 (2019).

4. Marquet K, Liesenborgs A, Bergs J, Vleugels A, Claes N. Incidence and outcome of inappropriate in-hospital empiric antibiotics for severe infection: a systematic review and meta-analysis. Critical Care, 19(1), 63 (2015).

5. Gandra S, Barter D, Laxminarayan R. Economic burden of antibiotic resistance: how much do we really know? Clinical Microbiology and Infection, 20(10), 973-979 (2014). ${ }^{*}$ Good study documenting the costs associated with AMR

6. Maragakis LL, Perencevich EN, Cosgrove SE. Clinical and economic burden of antimicrobial resistance. Expert review of anti-infective therapy, 6(5), 751-763 (2008). 
7. Aldeyab MA, Harbarth S, Vernaz $\mathrm{N}$ et al. The impact of antibiotic use on the incidence and resistance pattern of extended-spectrum beta-lactamase-producing bacteria in primary and secondary healthcare settings. British journal of clinical pharmacology, 74(1), 171-179 (2012).

8. Bartoloni A, Pallecchi L, Riccobono E et al. Relentless increase of resistance to fluoroquinolones and expanded-spectrum cephalosporins in Escherichia coli: 20 years of surveillance in resource-limited settings from Latin America. Clinical Microbiology and Infection, 19(4), 356-361 (2013).

9. Costelloe C, Metcalfe C, Lovering A, Mant D, Hay AD. Effect of antibiotic prescribing in primary care on antimicrobial resistance in individual patients: systematic review and meta-analysis. Bmj, 340, c2096 (2010).

10. Jinks $\mathrm{T}$, Lee $\mathrm{N}$, Sharland $\mathrm{M}$ et al. A time for action: antimicrobial resistance needs global response. Bulletin of the World Health Organization, 94(8), 558-558A (2016).

11. Saleem Z, Hassali MA. Travellers take heed: Outbreak of extensively drug resistant (XDR) typhoid fever in Pakistan and a warning from the US CDC. Travel medicine and infectious disease, (2018).

12. Founou RC, Founou LL, Essack SY. Clinical and economic impact of antibiotic resistance in developing countries: A systematic review and meta-analysis. PloS one, 12(12), e0189621 (2017).

13. O'Neill J. Securing new drugs for future generations: the pipeline of antibiotics. The review of antimicrobial resistance. Available at URL: https://amrreview.org/sites/default/files/SECURING\%20NEW\%20DRUGS\%20FOR\%20FUTURE\%20G ENERATIONS\%20FINAL\%20WEB_0.pdf.).

14. Falagas ME, Rafailidis PI. Re-emergence of colistin in today's world of multidrugresistant organisms: personal perspectives. Expert opinion on investigational drugs, 17(7), 973-981 (2008).

15. Karaaslan A, Çağan E, Kadayifci EK et al. Intravenous Colistin Use for Multidrug-Resistant Gram-Negative Infections in Pediatric Patients. Balkan Medical Journal, 33(6), 627 (2016).

16. Mendelson M, Brink A, Gouws J et al. The One Health stewardship of colistin as an antibiotic of last resort for human health in South Africa. The Lancet Infectious Diseases, (2018).

17. Sharland M, Pulcini C, Harbarth S et al. Classifying antibiotics in the WHO Essential Medicines List for optimal use-be AWaRe. The Lancet. Infectious diseases, 18(1), 18-20 (2018).

*Landmark classification of antibiotics

18. Slama TG, Amin A, Brunton SA et al. A clinician's guide to the appropriate and accurate use of antibiotics: the Council for Appropriate and Rational Antibiotic Therapy (CARAT) criteria. (Ed.^^(Eds) (Elsevier, 2005)

19. Fishman N. Policy statement on antimicrobial stewardship by the Society for Healthcare Epidemiology of America (SHEA), the Infectious Diseases Society of America (IDSA), and the Pediatric Infectious Diseases Society (PIDS). Infection Control \& Hospital Epidemiology, 33(4), 322-327 (2012). 
20. Zaidi S, Bigdeli M, Aleem N, Rashidian A. Access to essential medicines in Pakistan: policy and health systems research concerns. PloS one, 8(5), e63515 (2013).

21. Riaz H, Godman B, Hussain S et al. Prescribing of bisphosphonates and antibiotics in Pakistan: challenges and opportunities for the future. Journal of Pharmaceutical Health Services Research, 6(2), 111-121 (2015).

22. Saleem Z, Saeed H, Ahmad M et al. Antibiotic Self-Prescribing Trends, Experiences and Attitudes in Upper Respiratory Tract Infection among Pharmacy and Non-Pharmacy Students: A Study from Lahore. PloS one, 11(2), e0149929 (2016).

23. Khan MS, Ahmed Z, Jehan S, Khan S, Zaman S, Shah FU. Common trend of antibiotics usage in a tertiary care hospital of Peshawar, Pakistan. Journal of Ayub Medical College Abbottabad, 22(1), 118-120 (2010).

24. Atif M, Azeem M, Saqib A, Scahill S. Investigation of antimicrobial use at a tertiary care hospital in Southern Punjab, Pakistan using WHO methodology. Antimicrobial Resistance \& Infection Control, 6(1), 41 (2017).

25. Tasawer Baig M, Akbar Sial A, Huma A, Ahmed M, Shahid U, Syed N. Irrational antibiotic prescribing practice among children in critical care of tertiary hospitals. Pakistan journal of pharmaceutical sciences, 30 (2017).

26. Haque A, Hussain K, Ibrahim R et al. Impact of pharmacist-led antibiotic stewardship program in a PICU of low/middle-income country. BMJ Open Qual, 7(1), e000180 (2018).

27. Lusignani LS, Blacky A, Starzengruber P, Diab-Elschahawi APM, Wrba DIT, Presterl E. A national point prevalence study on healthcare-associated infections and antimicrobial use in Austria. Wiener klinische Wochenschrift, 128(3-4), 89-94 (2016).

28. Versporten A, Bielicki J, Drapier N et al. The Worldwide Antibiotic Resistance and Prescribing in European Children (ARPEC) point prevalence survey: developing hospitalquality indicators of antibiotic prescribing for children. Journal of Antimicrobial Chemotherapy, 71(4), 1106-1117 (2016).

29. Ansari F, Erntell M, Goossens H, Davey P, Group EIHCS. The European surveillance of antimicrobial consumption (ESAC) point-prevalence survey of antibacterial use in 20 European hospitals in 2006. Clinical infectious diseases, 49(10), 1496-1504 (2009).

30. Cotta M, Robertson M, Upjohn L, Marshall C, Liew D, Buising K. Using periodic pointprevalence surveys to assess appropriateness of antimicrobial prescribing in Australian private hospitals. Internal medicine journal, 44(3), 240-246 (2014).

31. Amadeo B, Zarb P, Muller A et al. European Surveillance of Antibiotic Consumption (ESAC) point prevalence survey 2008: paediatric antimicrobial prescribing in 32 hospitals of 21 European countries. Journal of antimicrobial chemotherapy, dkq309 (2010).

32. Xie D-s, Xiang L-I, Li R, Hu Q, Luo Q-q, Xiong W. A multicenter point-prevalence survey of antibiotic use in 13 Chinese hospitals. Journal of infection and public health, 8(1), 55-61 (2015).

33. Magill SS, Edwards JR, Beldavs ZG et al. Prevalence of antimicrobial use in US acute care hospitals, May-September 2011. Jama, 312(14), 1438-1446 (2014).

34. Usluer G, Ozgunes I, Leblebicioglu H. A multicenter point-prevalence study: antimicrobial prescription frequencies in hospitalized patients in Turkey. Annals of clinical Microbiology and Antimicrobials, 4(1), 16 (2005). 
35. Okoth C, Opanga S, Okalebo F, Oluka M, Baker Kurdi A, Godman B. Point prevalence survey of antibiotic use and resistance at a referral hospital in Kenya: findings and implications. Hospital Practice, 1-9 (2018).

36. Versporten A, Zarb P, Caniaux I et al. Antimicrobial consumption and resistance in adult hospital inpatients in 53 countries: results of an internet-based global point prevalence survey. The Lancet Global Health, 6(6), e619-e629 (2018).

${ }^{* *}$ Current results of the Global PPS study used for comparative purposes

37. Saleem Z, Hassali MA, Godman B, Hashmi FK, Saleem F. A multicenter point prevalence survey of health care-associated infections in Pakistan: Findings and implications. American journal of infection control, (2018).

${ }^{*}$ First comprehensive study of HAls in Pakistan

38. Global Point Prevalence Survey of Antimicrobial Consumption and Resistance (2018 GLOBAL-PPS). [cited 2018 July 23] Available at URL: http://www.global-pps.com/wpcontent/uploads/GLOBAL-PPS-2018-Protocol-1.pdf.)

39. Tiroyakgosi $C$, Matome $M$, Summers $E$ et al. Ongoing initiatives to improve the use of antibiotics in Botswana: University of Botswana symposium meeting report. Expert review of anti-infective therapy, 16(5), 381-384 (2018).

40. Nakwatumbah S, Kibuule D, Godman B et al. Compliance to guidelines for the prescribing of antibiotics in acute infections at Namibia's national referral hospital: a pilot study and the implications. Expert review of anti-infective therapy, 15(7), 713-721 (2017).

41. Niaz Q, Godman B, Massele A et al. Validity of World Health Organisation prescribing indicators in Namibia's primary healthcare: findings and implications. International journal for quality in health care : journal of the International Society for Quality in Health Care, (2018).

${ }^{*}$ Good study documenting the association of guideline adherence with the quality use of antibiotics

42. Organization $\mathrm{WH}$, Organization $\mathrm{WH}$. Collaborating centre for drug statistics methodology. Guidelines for ATC classification and DDD assignment, 3 (2009).

43. Khan EA. An urgent need for National Action Plan for Infection Control and Antibiotic Stewardship in Pakistan. Clin Infect Dis, 44, 159-177 (2007).

44. Versporten A, Sharland M, Bielicki J, Drapier N, Vankerckhoven V, Goossens H. The antibiotic resistance and prescribing in European Children project: a neonatal and pediatric antimicrobial web-based point prevalence survey in 73 hospitals worldwide. The Pediatric infectious disease journal, 32(6), e242-e253 (2013).

45. Zarb P, Amadeo B, Muller A et al. Identification of targets for quality improvement in antimicrobial prescribing: the web-based ESAC Point Prevalence Survey 2009. Journal of Antimicrobial Chemotherapy, 66(2), 443-449 (2011).

46. Okoth C, Opanga S, Okalebo F, Oluka M, Baker Kurdi A, Godman B. Point prevalence survey of antibiotic use and resistance at a referral hospital in Kenya: findings and implications. Hospital practice (1995), 46(3), 128-136 (2018).

47. Tiroyakgosi $\mathrm{C}$, Matome $\mathrm{M}$, Summers $\mathrm{E}$ et al. Ongoing initiatives to improve the use of antibiotics in Botswana: University of Botswana symposium meeting report. Expert review of anti-infective therapy, 16(5), 381-384 (2018). 
48. Labi A-K, Obeng-Nkrumah N, Nartey ET et al. Antibiotic use in a tertiary healthcare facility in Ghana: a point prevalence survey. Antimicrobial Resistance and Infection Control, 7, 15 (2018).

49. Ren $N$, Zhou $P$, Wen $X$ et al. Point prevalence survey of antimicrobial use in Chinese hospitals in 2012. American journal of infection control, 44(3), 332-339 (2016).

50. Lee CF, Cowling BJ, Feng $S$ et al. Impact of antibiotic stewardship programmes in Asia: a systematic review and meta-analysis. Journal of Antimicrobial Chemotherapy, 73(4), 844-851 (2018).

51. Zou X-x, Fang Z, Min R et al. Is nationwide special campaign on antibiotic stewardship program effective on ameliorating irrational antibiotic use in China? Study on the antibiotic use of specialized hospitals in China in 2011-2012. Journal of Huazhong University of Science and Technology [Medical Sciences], 34(3), 456-463 (2014).

52. Hou D, Wang Q, Jiang C, Tian C, Li H, Ji B. Evaluation of the short-term effects of antimicrobial stewardship in the intensive care unit at a tertiary hospital in China. PloS one, 9(7), e101447 (2014).

53. Phu VD, Wertheim HF, Larsson $M$ et al. Burden of hospital acquired infections and antimicrobial use in Vietnamese adult intensive care units. PloS one, 11(1), e0147544 (2016).

54. Ban KA, Minei JP, Laronga $C$ et al. American College of Surgeons and Surgical Infection Society: surgical site infection guidelines, 2016 update. Journal of the American College of Surgeons, 224(1), 59-74 (2017).

55. Gagliotti C, Balode A, Baquero F et al. Escherichia coli and Staphylococcus aureus: bad news and good news from the European Antimicrobial Resistance Surveillance Network (EARS-Net, formerly EARSS), 2002 to 2009. Eurosurveillance, (2011).

56. Meyer E, Schwab F, Schroeren-Boersch B, Gastmeier P. Dramatic increase of thirdgeneration cephalosporin-resistant E. coli in German intensive care units: secular trends in antibiotic drug use and bacterial resistance, 2001 to 2008. Critical care, 14(3), R113 (2010).

57. Clifford V, Daley A. Antibiotic prophylaxis in obstetric and gynaecological procedures: a review. Australian and New Zealand Journal of Obstetrics and Gynaecology, 52(5), 412 419 (2012).

58. Bratzler DW, Dellinger EP, Olsen KM et al. Clinical practice guidelines for antimicrobial prophylaxis in surgery. American journal of health-system pharmacy : AJHP : official journal of the American Society of Health-System Pharmacists, 70(3), 195-283 (2013).

59. Mwita JC, Souda S, Magafu M, Massele A, Godman B, Mwandri M. Prophylactic antibiotics to prevent surgical site infections in Botswana: findings and implications. Hospital practice (1995), 46(3), 97-102 (2018).

60. Berríos-Torres SI, Sarah HY, Bratzler DW et al. Activity of commonly used antimicrobial prophylaxis regimens against pathogens causing coronary artery bypass graft and arthroplasty surgical site infections in the United States, 2006-2009. Infection Control \& Hospital Epidemiology, 35(3), 231-239 (2014).

61. Stulberg JJ, Delaney CP, Neuhauser DV, Aron DC, Fu P, Koroukian SM. Adherence to surgical care improvement project measures and the association with postoperative infections. Jama, 303(24), 2479-2485 (2010). 
62. Harbarth S, Samore MH, Lichtenberg D, Carmeli Y. Prolonged antibiotic prophylaxis after cardiovascular surgery and its effect on surgical site infections and antimicrobial resistance. Circulation, 101(25), 2916-2921 (2000).

63. Vander Stichele R, Elseviers M, Ferech M, Blot S, Goossens H, Group † ESoACP. Hospital consumption of antibiotics in 15 European countries: results of the ESAC Retrospective Data Collection (1997-2002). Journal of Antimicrobial Chemotherapy, 58(1), 159-167 (2006).

64. Saleem Z, Hassali MA, Hashmi FK. Pakistan's national action plan for antimicrobial resistance: translating ideas into reality. The Lancet Infectious Diseases, 18(10), 10661067 (2018).

*Documenting the NAP of Pakistan

65. Ashiru-Oredope D, Sharland M, Charani E, McNulty C, Cooke J. Improving the quality of antibiotic prescribing in the NHS by developing a new Antimicrobial Stewardship Programme: Start Smart-Then Focus. Journal of antimicrobial chemotherapy, 67(suppl_1), i51-i63 (2012).

66. Sviestina I, Aston J, Mozgis D. Comparison of antimicrobial prescribing between two specialist paediatric centres in the UK and Latvia. European Journal of Hospital Pharmacy: Science and Practice, ejhpharm-2012-000179 (2013).

67. Fitzpatrick RW, Edwards CM. Evaluation of a tool to benchmark hospital antibiotic prescribing in the United Kingdom. Pharmacy World \& Science, 30(1), 73-78 (2008).

68. Cox JA, Vlieghe $\mathrm{E}$, Mendelson $\mathrm{M}$ et al. Antibiotic stewardship in low- and middle-income countries: the same but different? Clinical microbiology and infection : the official publication of the European Society of Clinical Microbiology and Infectious Diseases, 23(11), 812-818 (2017).

${ }^{* *}$ Good study documenting the challenges with ASPs in LMICs

69. Zarb P, Goossens H. European surveillance of antimicrobial consumption (ESAC). Drugs, 71(6), 745-755 (2011).

70. Robert J, Pean Y, Varon E et al. Point prevalence survey of antibiotic use in French hospitals in 2009. Journal of antimicrobial chemotherapy, dkr571 (2012).

71. Jabeen K, Zafar A, Hasan R. Frequency and sensitivity pattern of extended spectrum beta lactamase producing isolates in a tertiary care hospital laboratory of Pakistan. Journal of Pakistan Medical Association, 55(10), 436 (2005).

72. Reynolds L, McKee M. Factors influencing antibiotic prescribing in China: an exploratory analysis. Health Policy, 90(1), 32-36 (2009).

73. Md Rezal RS, Hassali MA, Alrasheedy AA, Saleem F, Md Yusof FA, Godman B. Physicians' knowledge, perceptions and behaviour towards antibiotic prescribing: a systematic review of the literature. Expert review of anti-infective therapy, 13(5), 665-680 (2015).

74. Jelinek GA, Neate SL. The influence of the pharmaceutical industry in medicine. Journal of law and medicine, 17(2), 216-223 (2009).

75. Ogunleye OO, Fadare JO, Yinka-Ogunleye AF, Anand Paramadhas BD, Godman B. Determinants of antibiotics prescribing among doctors in a Nigerian urban tertiary hospital. Hospital Practice, (just-accepted) (2018).

76. Civaner M. Sale strategies of pharmaceutical companies in a "pharmerging" country: The problems will not improve if the gaps remain. Health policy, 106(3), 225-232 (2012). 
77. Reynolds L, McKee M. Factors influencing antibiotic prescribing in China: an exploratory analysis. Health policy, 90(1), 32-36 (2009).

78. Cyriac JM, James E. Switch over from intravenous to oral therapy: A concise overview. Journal of pharmacology \& pharmacotherapeutics, 5(2), 83 (2014).

79. Lorgelly PK, Atkinson M, Lakhanpaul $\mathrm{M}$ et al. Oral versus iv antibiotics for communityacquired pneumonia in children: a cost-minimisation analysis. European Respiratory Journal, 35(4), 858-864 (2010).

80. Magedanz L, Silliprandi EM, dos Santos RP. Impact of the pharmacist on a multidisciplinary team in an antimicrobial stewardship program: a quasi-experimental study. Int J Clin Pharm, 34(2), 290-294 (2012).

81. Zhang H-X, Li X, Huo H-Q, Liang P, Zhang J-P, Ge W-H. Pharmacist interventions for prophylactic antibiotic use in urological inpatients undergoing clean or cleancontaminated operations in a Chinese hospital. PloS one, 9(2), e88971 (2014).

${ }^{*}$ Good study documenting the impact of pharmacist interventions with improving antibiotic use

82. Antonioli P, Manzalini MC, Stefanati A et al. Temporal trends of healthcare associated infections and antimicrobial use in 2011-2013, observed with annual point prevalence surveys in Ferrara University Hospital, Italy. Journal of Preventive Medicine and Hygiene, 57(3), E135 (2016). 
Table 1: Overall antibiotic use prevalence

\begin{tabular}{|c|c|c|c|c|c|c|c|c|c|c|c|c|c|c|}
\hline Characteristics N (\%) & Hosp. $A^{i}$ & Hosp. B & Hosp. C & Hosp. D & Hosp. $\mathrm{E}^{\mathrm{ii}}$ & Hosp. $F^{\text {iii }}$ & Hosp. $G^{\text {ii }}$ & Hosp. H & Hosp. I & Hosp. J & Hosp. K & Hosp. L & Hosp. M & Total \\
\hline Hospital Type & Public & Charity & Public & Private & Charity & Charity & Public & Public & Public & Private & Public & Public & Public & \\
\hline Total beds & 451 & $233^{\circ}$ & 86 & 67 & 190 & 195 & 361 & 166 & 107 & 239 & 282 & 355 & 342 & 3074 \\
\hline Hospitalized patients & $373(82.7)$ & $102(43.7)$ & $72(83.7)$ & $36(53.7)$ & $93(48.9)$ & $161(82.6)$ & $212(58.7)$ & $96(57.8)$ & $75(70.1)$ & $106(44.4)$ & $112(39.7)$ & $286(80.6)$ & $230(67.3)$ & 1954 (63.6) \\
\hline Number of treated patients & $281(75.3)$ & $97(95.0)$ & $68(94.4)$ & $27(75.0)$ & $65(69.8)$ & $82(50.9)$ & $148(69.8)$ & $79(82.3)$ & $75(100)$ & $89(84.0)$ & $74(66.1)$ & $230(80.4)$ & $201(87.4)$ & $1516(77.6)$ \\
\hline $\begin{array}{l}\text { Prescribed antibiotics (per } \\
\text { patient) }\end{array}$ & 481 & 149 & 122 & 51 & 108 & 140 & 291 & 105 & 127 & 134 & 84 & 338 & 353 & 2483 \\
\hline Surgical Department N (\%) & $267(55.5)$ & $48(32.2)$ & $73(59.8)$ & $38(74.5)$ & $61(56.5)$ & $19(13.6)$ & $73(25.1)$ & $5(4.8)$ & $73(57.5)$ & $23(17.2)$ & $48(57.1)$ & $182(53.8)$ & $85(24.1)$ & $961(38.7)$ \\
\hline Medical Department N (\%) & $208(43.2)$ & $98(65.8)$ & $49(40.2)$ & $4(7.8)$ & $40(37.0)$ & $105(75.0)$ & $199(68.4)$ & $97(92.4)$ & $54(42.5)$ & $72(53.7)$ & $23(27.4)$ & $156(46.2)$ & $265(75.1)$ & $1404(56.5)$ \\
\hline Intensive Care Unit N (\%) & $6(1.2)$ & $3(2.0)$ & 0 & $9(17.6)$ & $7(6.5)$ & $16(11.4)$ & $19(6.5)$ & $3(2.9)$ & 0 & $39(29.1)$ & $13(15.5)$ & 0 & $3(0.8)$ & $118(4.8)$ \\
\hline Male & $172(35.8)$ & $63(42.3)$ & $57(46.7)$ & $33(64.7)$ & $53(49.1)$ & $84(60.0)$ & $104(35.7)$ & $62(59.0)$ & $62(48.8)$ & $67(50.0)$ & $33(39.3)$ & $178(52.7)$ & $209(59.2)$ & $1177(47.4)$ \\
\hline Female & $309(64.2)$ & $86(57.7)$ & $65(53.3)$ & $18(35.3)$ & $55(50.9)$ & $56(40.0)$ & $187(64.3)$ & $43(41.0)$ & $65(51.2)$ & $67(50.0)$ & $51(60.7)$ & $160(47.3)$ & $144(40.8)$ & $1306(52.6)$ \\
\hline \multicolumn{15}{|l|}{ Route of administration } \\
\hline Oral & $31(6.4)$ & $18(12.1)$ & 0 & $2(3.9)$ & $15(13.9)$ & $25(17.9)$ & $15(5.2)$ & $2(1.9)$ & $9(7.1)$ & $25(18.7)$ & $24(28.6)$ & $30(8.9)$ & $13(3.7)$ & $209(8.4)$ \\
\hline Parenteral & $450(93.6)$ & $131(87.9)$ & $122(100)$ & $49(96.1)$ & $93(86.1)$ & $115(82.1)$ & $276(94.8)$ & $103(98.1)$ & $118(92.9)$ & $109(81.3)$ & $60(71.4)$ & $308(91.1)$ & $340(96.3)$ & $2274(91.5)$ \\
\hline \multicolumn{15}{|l|}{ Indication } \\
\hline Therapeutic use & $124(25.8)$ & $57(38.3)$ & $37(30.3)$ & $16(31.3)$ & $42(38.9)$ & $106(75.7)$ & $112(38.5)$ & $26(24.8)$ & $59(46.5)$ & $75(56.0)$ & $36(42.9)$ & $111(32.8)$ & $151(42.8)$ & $997(40.2)$ \\
\hline Prophylaxic use & $351(73.0)$ & $83(55.7)$ & $84(68.9)$ & $31(60.6)$ & $66(61.1)$ & $34(25.3)$ & $154(52.9)$ & $76(72.4)$ & $68(53.5)$ & $54(40.3)$ & $48(57.1)$ & $226(66.9)$ & $197(55.8)$ & $1426(57.4)$ \\
\hline Unknown & $6(1.2)$ & $9(6.0)$ & $1(0.8)$ & $4(7.8)$ & 0 & 0 & $25(8.6)$ & $3(2.9)$ & 0 & $5(3.7)$ & 0 & $1(0.3)$ & $5(1.4)$ & $59(2.4)$ \\
\hline \multicolumn{15}{|l|}{ Indication for prophylaxis } \\
\hline Medical & $110(31.3)$ & $33(39.7)$ & $25(29.7)$ & $8(25.8)$ & $18(27.3)$ & $18(52.9)$ & $34(77.9)$ & $54(71.1)$ & $26(38.2)$ & $30(55.5)$ & $18(37.5)$ & $78(34.5)$ & $81(53.6)$ & $533(37.4)$ \\
\hline Surgical & $241(68.6)$ & $50(61.3)$ & $59(70.3)$ & $23(74.2)$ & $48(73.7)$ & $16(47.1)$ & $120(22.1)$ & $22(28.9)$ & $42(61.8)$ & $24(44.5)$ & $30(62.5)$ & $148(65.5)$ & $70(46.3)$ & $893(62.6)$ \\
\hline \multicolumn{15}{|l|}{ Surgical prophylaxis } \\
\hline Single dose & 0 & $3(6.0)$ & 0 & 0 & 0 & 0 & 0 & 0 & $2(4.8)$ & $1(4.2)$ & 0 & $4(2.7)$ & 0 & $10(1.1)$ \\
\hline One day & $5(2.1)$ & 0 & $3(5.1)$ & 0 & $1(2.1)$ & 0 & $1(0.8)$ & 0 & 0 & 0 & 0 & $3(2.0)$ & 0 & $13(1.5)$ \\
\hline More than one day & $236(97.9)$ & $47(94.0)$ & $56(94.9)$ & $23(100)$ & $47(97.9)$ & $16(100)$ & $119(99.2)$ & $22(100)$ & $40(95.2)$ & $23(95.8)$ & $30(100)$ & $141(95.3)$ & 70 & $870(97.4)$ \\
\hline \multicolumn{15}{|l|}{ Indication for infec } \\
\hline Community-acquired & $116(93.5)$ & $53(93.0)$ & $31(83.7)$ & $9(56.3)$ & $32(76.2)$ & $69(65.1)$ & $105(93.7)$ & $22(84.6)$ & $55(93.2)$ & $45(60.8)$ & $27(75.0)$ & $99(89.2)$ & $187(94.9)$ & $850(85.3)$ \\
\hline Hospital-acquired & $8(6.5)$ & $4(7.0)$ & $6(16.3)$ & $7(43.7)$ & $10(24.8)$ & $37(34.9)$ & $7(6.3)$ & $4(15.4)$ & $4(6.8)$ & $29(39.2)$ & $9(25.0)$ & $12(10.8)$ & $10(5.1)$ & $147(14.7)$ \\
\hline \multicolumn{15}{|l|}{ Reason on Notes } \\
\hline Yes & $88(18.3)$ & $25(16.8)$ & $11(9.0)$ & $4(7.8)$ & $3(2.8)$ & $78(55.7)$ & $25(8.6)$ & $15(14.3)$ & $44(34.6)$ & $47(35.1)$ & $16(19.0)$ & $87(25.7)$ & $147(41.6)$ & $590(23.8)$ \\
\hline No & $393(81.7)$ & $124(83.2)$ & $111(91.0)$ & $47(92.2)$ & $105(97.2)$ & $62(44.3)$ & $266(91.4)$ & $90(85.7)$ & $83(65.4)$ & $87(64.9)$ & $68(81.0)$ & $251(74.3)$ & $206(58.4)$ & $1893(76.2)$ \\
\hline Empirical Therapy & $480(99.8)$ & $148(99.3)$ & $122(100)$ & $48(94.1)$ & $104(96.3)$ & $92(65.7)$ & $285(97.9)$ & $104(99.0)$ & 127 & $107(79.9)$ & $84(100.0)$ & $335(99.1)$ & $353(100.0)$ & $2389(96.2)$ \\
\hline rics used & 22 & 15 & 9 & 14 & 23 & 26 & 29 & 15 & 13 & 30 & 11 & 22 & 23 & 19 \\
\hline Culture Reports & 1 & 1 & 0 & 3 & 4 & 22 & 1 & 0 & 0 & 25 & 0 & 0 & 0 & 57 \\
\hline
\end{tabular}

${ }^{i}$ Public Sector Teaching Hospital, ${ }^{\text {i }}$ Specialized Spine and Orthopedic Hospital, ${ }^{\text {iii }}$ Specialized Cancer Care Hospital with advance Clinical Pharmacy Services 
Table 2: Use prevalence of main antibiotics classes

\begin{tabular}{llc}
\hline Antibiotics & N (\%) & $\begin{array}{l}\text { Hospital range } \\
\text { \% }\end{array}$ \\
\hline ANTIBACTERIALS FOR SYSTEMIC USE (J01) & $2398(96.6)$ & $85.0-100.0$ \\
Tetracyclines (J01A) & $23(0.8)$ & $0.0-5.9$ \\
Penicillins (J01C) & $293(11.8)$ & $0.0-35.0$ \\
Cephalosporins \& Penams (J01D) & $1218(49.1)$ & $0.0-70.2$ \\
Sulfonamides \& trimethoprim (J01E) & $5(0.2)$ & $0.0-2.9$ \\
Macrolides \& lincosamides (J01F) & $51(2.1)$ & $0.0-5.2$ \\
Aminoglycosides (J01G) & $88(3.5)$ & $0.0-11.9$ \\
Quinolones (J01M) & $282(11.4)$ & $0.0-24.6$ \\
Other antibacterials (J01X) & $458(18.4)$ & $0.0-31.1$ \\
ANTIMYCOTICS FOR SYSTEMIC USE(J02) & $11(0.4)$ & $0.0-7.1$ \\
ANTIMYCOBACTERIALS FOR SYSTEMIC USE (J04) & $21(0.8)$ & $0.0-6.5$ \\
ANTIVIRALS FOR SYSTEMIC USE (J05) & $21(0.8)$ & $0.0-3.6$ \\
ANTIPROTOZOALS (P01) & $15(0.6)$ & $0.0-3.6$ \\
ANTIDIARRHEALS (A07) & $17(0.7)$ & $0.0-4.5$ \\
\hline
\end{tabular}

Table 3: Top 10 indications and antibiotics

\begin{tabular}{|c|c|c|c|c|}
\hline \multirow{2}{*}{ No. } & \multicolumn{2}{|c|}{ Top 10 Indications } & \multicolumn{2}{|c|}{ Top 10 Antibiotics } \\
\hline & Indications & $\mathbf{N}(\%)$ & Antibiotics & $\mathbf{N}(\%)$ \\
\hline 1. & P. OBGY & $408(16.5)$ & Ceftriaxone & $868(35.0)$ \\
\hline 2. & P. GI & $313(12.6)$ & Metronidazole & $397(16.0)$ \\
\hline 3. & LRTI & $297(12.0)$ & Ciprofloxacin & $150(6.0)$ \\
\hline 4. & General MP & $260(10.5)$ & Co-amoxiclav & $91(3.7)$ \\
\hline 5. & SST & $170(6.8)$ & Moxifloxacin & $86(3.5)$ \\
\hline 6. & P. BJ & $149(6.0)$ & Piperacillin, enzyme inhibitor & $82(3.3)$ \\
\hline 7. & SEPSIS & $145(5.9)$ & Cefoperazone & $73(2.9)$ \\
\hline 8. & GIT & $118(4.8)$ & Amikacin & $69(2.8)$ \\
\hline 9. & P. RESP & $95(3.8)$ & Ampicillin & $68(2.7)$ \\
\hline 10. & CNS & $62(2.5)$ & Vancomycin & $59(2.4)$ \\
\hline
\end{tabular}

BJ; Bone \& Joint, CNS; Central Nervous System, GIT; Gastro-Intestinal Tract, MP; Medical Prophylaxis, OBGY; Obstetric or Gynaecological, LRTI; Lower Respiratory Tract Infection, P; Medical or Surgical Prophylaxis, RESP; Respiratory, SST; Skin and Soft Tissues 
Table 4: Top 5 antibiotics in different conditions

\begin{tabular}{|c|c|c|c|c|c|}
\hline \multirow[t]{2}{*}{ Study Variables } & \multicolumn{5}{|c|}{ Ranks } \\
\hline & 1 & 2 & 3 & 4 & 5 \\
\hline \multicolumn{6}{|l|}{ DEPARTMENT } \\
\hline \multirow[t]{2}{*}{ ICU } & Ceftriaxone & Amikacin & Meropanam & Piperacillin \& & Vancomycin \\
\hline & $15(12.7)$ & $13(11.0)$ & $12(10.2)$ & $\begin{array}{l}\text { enzyme inhibitor } \\
12(10.2)\end{array}$ & $11(9.3)$ \\
\hline \multirow{2}{*}{ Medical } & Ceftriaxone & Ciprofloxacin & Piperacillin \& & Moxifloxacin & Ampicillin \\
\hline & $567(40.4)$ & $58(4.1)$ & $\begin{array}{l}\text { enzyme inhibitor } \\
58(4.1)\end{array}$ & $57(4.1)$ & $52(3.7)$ \\
\hline \multirow[t]{2}{*}{ Surgical } & Ceftriaxone & Metronidazole & Cefoperazone & Co-amoxiclav & Cefotaxime \\
\hline & $286(29.8)$ & $218(22.7)$ & $66(6.9)$ & $47(4.9)$ & $42(4.4)$ \\
\hline \multicolumn{6}{|l|}{ AGE GROUPS } \\
\hline \multirow[t]{2}{*}{$<1$ month } & Ceftriaxone & Amikacin & Cefotaxime & Ampicillin & Ciprofloxacin \\
\hline & $16(23.5)$ & $11(16.2)$ & $11(16.2)$ & $6(8.8)$ & $4(5.9)$ \\
\hline \multirow[t]{2}{*}{1 month-1year } & Ceftriaxone & Ampicillin & Metronidazole & Amikacin & Ceftazidime \\
\hline & $70(44.0)$ & $33(20.8)$ & $10(6.3)$ & $7(4.4)$ & $6(3.8)$ \\
\hline \multirow[t]{2}{*}{1 year-25years } & Ceftriaxone & Metronidazole & Cefotaxime & Ciprofloxacin & Co-amoxiclav \\
\hline & $228(34.6)$ & $127(19.3)$ & $32(4.9)$ & $27(4.1)$ & $27(4.1)$ \\
\hline \multirow{2}{*}{ 26-65years } & Ceftriaxone & Metronidazole & Ciprofloxacin & Moxifloxacin & Co-amoxiclav \\
\hline & $471(34.2)$ & $241(17.5)$ & $111(8.1)$ & $61(4.4)$ & $57(4.1)$ \\
\hline \multirow[t]{2}{*}{$>65$ years } & Ceftriaxone & Moxifloxacin & Metronidazole & Meropenam & Piperacillin \& \\
\hline & $83(37.6)$ & $20(9.0)$ & $19(8.6)$ & $13(5.9)$ & $\begin{array}{l}\text { enzyme inhibitor } \\
10(4.5)\end{array}$ \\
\hline \multicolumn{6}{|l|}{ INDICATIONS } \\
\hline \multirow{2}{*}{$\begin{array}{l}\text { Community acquired } \\
\text { infection }\end{array}$} & Ceftriaxone & Metronidazole & Ampicillin & Vancomycin & Moxifloxacin \\
\hline & $237(27.9)$ & 95 (11.2) & $43(5.1)$ & $40(4.7)$ & $40(4.7)$ \\
\hline \multirow{2}{*}{$\begin{array}{l}\text { Hospital acquired } \\
\text { infection }\end{array}$} & Ceftriaxone & Piperacillin \& & Metronidazole & Meropenam & Amikacin \\
\hline & $25(16.9)$ & $\begin{array}{l}\text { enzyme inhibitor } \\
16(10.8)\end{array}$ & $13(8.8)$ & $9(6.1)$ & $9(6.1)$ \\
\hline \multirow[t]{2}{*}{ Medical Prophylaxis } & Ceftriaxone & Metronidazole & Ciprofloxacin & Moxifloxacin & Piperacillin \& \\
\hline & $278(52.2)$ & $43(8.1)$ & $31(5.8)$ & $24(4.5)$ & $\begin{array}{l}\text { enzyme inhibitor } \\
18(3.4)\end{array}$ \\
\hline \multirow[t]{2}{*}{ Surgical Prophylaxis } & Ceftriaxone & Metronidazole & Ciprofloxacin & Cefoperazone & Cefotaxime \\
\hline & $310(34.7)$ & $238(26.7)$ & $63(7.1)$ & $54(6.0)$ & $42(4.7)$ \\
\hline \multirow[t]{2}{*}{ Unknown/others } & Ceftriaxone & Metronidazole & Ciprofloxacin & Levofloxacin & Co-amoxiclav \\
\hline & $18(30.5)$ & $8(13.6)$ & $5(8.5)$ & $3(5.1)$ & $3(5.1)$ \\
\hline
\end{tabular}

